\title{
$S$-образные вольт-амперные характеристики мощных диодов Шоттки при больших плотностях тока
}

\author{
(C) А.Г. Тандоев, Т.Т. Мнацаканов ", С.Н. Юрков \\ Национальный исследовательский университет „Московский энергетический институт“, \\ 111250 Москва, Россия \\ ฯ E-mail: mnatt@yandex.ru
}

Поступила в Редакцию 24 декабря 2019 г.

В окончательной редакции 28 декабря 2019 г.

Принята к публикации 30 декабря 2019 г.

\begin{abstract}
Проведен последовательный учет влияния совокупности квазинейтральных режимов переноса носителей заряда в полупроводниках, включающей в себя наряду с диффузией и дрейфом обнаруженный недавно режим диффузии, стимулированной квазинейтральным дрейфом (DSQD). Исследован порядок смены режимов переноса носителей заряда в структурах диода Шоттки, и показано, к каким особенностям вольт-амперных характеристик это может приводить. Результаты аналитического исследования особенностей проверены и подтверждены с помощью численного моделирования.
\end{abstract}

Ключевые слова: перенос носителей заряда в полупроводниках, мощные диоды Шоттки, вольт-амперная характеристика, режимы переноса носителей.

DOI: 10.21883/FTP.2020.05.49264.9339

\section{1. Введение}

В настоящее время диоды Шоттки (ДШ) являются одними из наиболее распространенных приборов полупроводниковой электроники. Мощные ДШ применяются при создании устройств компенсации реактивной мощности, коммутаторов солнечных батарей, в автомобильной электронике. Отсутствие накопления неосновных носителей заряда в базовой области ДШ в стандартных рабочих режимах при протекании прямого тока определяет преимущества ДШ перед приборами с $p-n$-переходами по ряду важных динамических параметров - быстродействию, коммутационным потерям и т. д. Вместе с тем следует иметь в виду, что в аварийных режимах, например, в режиме короткого замыкания или в режиме ударного тока, плотность прямого тока может превосходить плотность тока в стандартном рабочем режиме в десятки раз [1-3]. Это может приводить к особенностям в работе ДШ. В ряде первых работ, посвященных исследованию ДШ, отмечались не только возможность инжекции неосновных носителей заряда контактом Шоттки при достаточно большом прямом смещении на нем, но и протекание прямого тока в режиме высокого уровня инжекции (ВУИ) в базовой области [4-6]. Наиболее полное исследование процессов при протекании прямого тока в условиях ВУИ, проведенное в рамках диффузионного приближения, представлено в работе [7].

Интерес к поведению ДШ в присутствии неосновных носителей заряда в базовой области возник вновь с появлением ДШ на основе карбида кремния. Казалось бы, большая ширина запрещенной зоны карбида кремния должна исключить появление этих носителей, так как они появляются, если высота барьера Шоттки превышает половину ширины запрещенной зоны $E_{g}$. Однако в работе [8] отмечалось появление неосновных носителей заряда в базовой области ДШ на основе 4H-SiC. Возможно, этот результат связан с появлением и быстрым распространением JBS-структур (junction barrier Schottky diodes). Появление таких структур вновь вызвало интерес к влиянию неосновных носителей заряда на характеристики этих приборов. JBS-приборы представляют собой интегрированные структуры, в которых области Ме- $n$ (Ме - металл) перемежаются с $p-n$-областями [9]. В настоящее время большинство ДШ выполнено в виде JBS-структур [10].

Протекание прямого тока через JBS-структуру качественно можно описать как протекание тока через параллельное соединение ДШ и диодной структуры с $p-n$-переходом [11]. В работах [12,13] были рассмотрены вольт-амперные характеристики (BAX) диодных структур с $p$-n-переходами при больших плотностях токов, когда в базовой области n-типа проводимости перенос носителей заряда осуществлялся в режиме ВУИ. Было показано, что, если отношение толщины базового слоя $W_{n}$ к амбиполярной диффузионной длине $L$ достигает величины $\sim(8-10)$, то наряду с хорошо известными квазинейтральными режимами переноса носителей заряда (диффузионным и дрейфовым) необходимо учитывать режим диффузии, стимулированной квазинейтральным дрейфом (DSQD mode). Учет этого режима оказывает существенное влияние на вид прямой BAX. В работе [12] было показано, что режим DSQD в базовой области может появиться и при малой величине отношения $W_{n} / L$, если распределение концентрации носителей заряда в базовой области сильно перекошено. Такая ситуация характерна для ДШ, так как в ДШ наблюдается существенная асимметрия инжектирующих способностей переходов Ме-п/п (металл-полупроводник) и $n-n^{+}$. 
Недавно в работах $[14,15]$ был представлен аналитический расчет ВАХ ДШ при плотностях тока, когда во всей базовой области был реализован режим ВУИ. В обеих работах было учтено влияние области DSQD на вид BAX. Это приводило к парадоксальной зависимости величины падения напряжения от уровня легирования базовой области $N_{d}$. Если во всей базовой области осуществлялся режим ВУИ, то с ростом $N_{d}$ падение напряжения увеличивалось. Расчет проводился для значений $W_{n} / L<1$.

В настоящей работе исследуется влияние величины отношения $W_{n} / L$ на вид ВАХ ДШ при ВУИ в базовой области $n$-типа проводимости. Отметим, что величина этого отношения может возрасти и стать $>1$ в результате, например, радиационного воздействия. Будет показано, что это может приводить к немонотонности распределения концентрации неравновесных носителей заряда и появлению на распределении точки минимума. В результате в базовой области ДШ кроме областей, в которых реализуются диффузионный перенос и перенос в режиме DSQD [14,15], появится еще и область, примыкающая к контакту Шоттки, в которой осуществляется режим квазинейтрального дрейфового переноса (QND). Вследствие существенной асимметрии инжектирующей способности контакта $\mathrm{Me}-п / п$ и $n-n^{+}$-перехода с ростом плотности тока $j$ точка минимума на распределении носителей заряда смещается в сторону перехода Ме-п/п и при некоторой плотности тока $j_{s t}$ совмещается с точкой $x=0$. При этом распределение носителей становится монотонным и сопровождается исчезновением области, в которой перенос носителей заряда осушествлялся в режиме QND. Такое перестроение распределения концентрации неравновесных носителей заряда приводит к перестроению квазинейтрального электрического поля и к появлению особенностей на ВАХ ДШ. Результаты аналитического исследования этих особенностей будут проверены и подтверждены с помощью численного моделирования.

\section{2. Постановка задачи}

Рассмотрим ДШ с базовой областью $n$-типа проводимости, в которой $W_{n} / L>1$. На рис. 1 схематичести представлена рассматриваемая структура. Начало координат $x=0$ выбрано на границе квазинейтральной части базы и области пространственного заряда контакта Ме-п/п. Будем рассматривать такие плотности прямого тока, при которых во всей базовой области шириной $W_{n}$ реализуется ВУИ.

Для расчета ВАХ ДШ необходимо решить уравнение непрерывности при ВУИ в базовом слое с граничными условиями, которые были ранее успешно использованы в работах $[14,15]$ :

$$
\frac{d^{2} p}{d x^{2}}-\frac{j}{j_{d}}\left(1-\frac{j}{j_{c r}}\right) \frac{N_{d}^{2}}{p^{2} L} \frac{d p}{d x}=\frac{p}{L^{2}},
$$

$$
\begin{gathered}
\left.\frac{d p}{d x}\right|_{x=0}=-\frac{j}{2 q D_{p}}+\frac{j_{s 0}}{q D} \frac{p^{2}(0)}{n_{i}^{2}}-\frac{j}{j_{d}}\left(1-\frac{j}{j_{c r}}\right) \frac{N_{d}^{2}}{p(0) L} \\
\left.\frac{d p}{d x}\right|_{x=W_{n}}=\frac{j}{2 q D_{p}}-\frac{j_{s p}}{q D} \frac{p^{2}\left(W_{n}\right)}{n_{i}^{2}}
\end{gathered}
$$

где

$$
\begin{gathered}
j_{d}=\frac{2 q D_{p}(b+1) N_{d}}{L}, \quad j_{c r}=\frac{q\left(\mu_{n 0}+\mu_{p 0}\right) N_{d}}{\left(1 / F_{s n}-1 / F_{s p}\right)}, \\
L=\sqrt{\frac{2 b}{b+1} D_{p} \tau}
\end{gathered}
$$

- амбиполярная диффузионная длина, $\mu_{n 0}$ и $\mu_{p 0}-$ подвижности электронов и дырок в слабом электрическом поле, $F_{s n}$ и $F_{s p}-$ характерные значения электрического поля, при которых дрейфовые скорости электронов и дырок насыщаются, $j_{s 0}=A^{*} T^{2} \exp \left(-q \varphi_{B 0} / k T\right)-$ плотность тока насыщения перехода $\mathrm{Me}-\Pi / п, A^{*}$ - эффективная постоянная Ричардсона, $j_{s p}-$ плотность тока насыщения $n^{+}-n$-перехода.

Так как плотность тока насыщения перехода Ме-п/п $j_{s 0}$ на 4-5 порядков больше плотности тока насыщения $n-n^{+}$-перехода $j_{s p}$, то концентрация неравновесных носителей заряда вблизи перехода $\mathrm{Me}-\Pi / п ~ p(0)$ значительно меньше величины $p(W)$ - концентрации неравновесных носителей заряда вблизи $n-n^{+}$-перехода.

В работах $[14,15]$ был рассмотрен случай, когда величина $W_{n} / L<1$ и концентрация неравновесных носителей заряда $p(x)$ монотонно возрастала от анодного перехода к катодному. Если же $W_{n} / L>1$, например $W_{n} / L=1.5$, то распределение концентрации неравновесных носителей заряда $p(x)$ перестает быть монотонно возрастающим и имеет точку минимума при плотности тока, не превышающей некоторую величину $j_{s t}$, которая

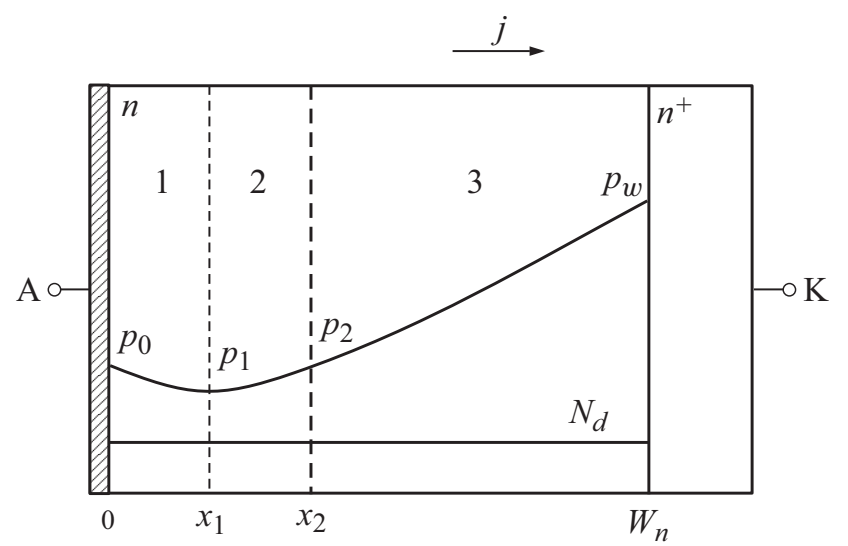

Рис. 1. Схема распределения носителей заряда в рассматриваемой структуре диода Шоттки. Левее точки $x_{1}$ располагается область 1 квазинейтрального дрейфового переноса (область QND). Правее точки $x_{1}$ располагается область 2 диффузии, стимулированной квазинейтральным дрейфом (область DSQD). В области 3 , примыкающей к $n-n^{+}$-переходу, превалирует диффузионное приближение переноса носителей заряда. A, $\mathrm{K}$ - анод и катод соответственно. 
будет определена далее. Пусть точка $x_{1}$ - координата точки минимума распределения $p(x)$, а $p_{1}=p\left(x_{1}\right)-$ минимальная концентрация неравновесных носителей заряда при плотности тока $j<j_{s t}$. Если плотность тока $j$ возрастает и приближается к величине $j_{s t}$, то координата $x_{1}$ точки минимума приближается к переходу $\mathrm{Me}-\Pi / \Pi$, т.е. к началу координат. При плотности тока, превышающей величину $j_{s t}$, распределение неравновесных носителей заряда становится монотонно возрастающим во всей базовой области. Найдем условие, при выполнении которого $j_{s t}$ не превышает величину $j_{c r}$. При плотности тока $j=j_{c r}$ решение уравнения непрерывности (1) с граничными условиями (2) и (3) приобретает стандартный диффузионный вид

$$
p(x)=a e^{x / L}+b e^{-x / L}
$$

с граничными концентрациями

$$
\begin{gathered}
\left.p_{0}\right|_{j_{c r}}=\left.p(0)\right|_{j_{c r}}=n_{i} \sqrt{\frac{b}{b+1} \frac{j_{c r}}{j_{s 0}}}, \\
\left.p_{w}\right|_{j_{c r}}=\left.p\left(W_{n}\right)\right|_{j_{c r}}=n_{i} \sqrt{\frac{b}{b+1} \frac{j_{c r}}{j_{s p}}} .
\end{gathered}
$$

Координата точки минимума $x_{1}$ удовлетворяет очевидному условию

$$
\left.\frac{d p}{d x}\right|_{x_{1}}=0
$$

Монотонность возрастания концентрации неравновесных носителей заряда $p(x)$ обеспечивается требованием

$$
\left.x_{1}\right|_{j_{c r}} \leq 0
$$

Элементарные вычисления приводят к условию

$$
\begin{aligned}
\frac{W_{n}}{L} \leq\left(\frac{W_{n}}{L}\right)_{m} & =\operatorname{arch} \frac{\left.p_{w}\right|_{j_{c r}}}{\left.p_{0}\right|_{j_{c r}}} \\
& \equiv \ln \left[\frac{\left.p_{w}\right|_{j_{c r}}}{\left.p_{0}\right|_{j_{c r}}}+\sqrt{\left(\frac{\left.p_{w}\right|_{j_{c r}}}{\left.p_{0}\right|_{j_{c r}}}\right)^{2}-1}\right] .
\end{aligned}
$$

Таким образом, если $W_{n} / L<\left(W_{n} / L\right)_{m}$, то при плотности тока $j=j_{c r}$ распределение концентрации неравновесных носителей заряда $p(x)$ уже станет монотонно возрастающим. Следовательно, величина $j_{s t}$ будет меньше, чем $j_{c r}$.

Оценим величину $\left(W_{n} / L\right)_{m}$ при $j_{s 0}=10^{-7} \mathrm{~A} / \mathrm{cm}^{2}$, $j_{s p}=10^{-12} \mathrm{~A} / \mathrm{cm}^{2}$ и $b=3$. Оказалось, что при этих значениях параметров $\left(W_{n} / L\right)_{m}=5.9$. В данной работе рассматриваются структуры, в которых отношение $W_{n} / L \leq 1.6$, и полученное условие выполняется.

Рассмотрим возможные случаи. В интервале плотностей тока от величины $j_{h}$, когда во всей базовой области реализуется режим ВУИ, до величины $j_{s t}<j_{c r}$ распределение концентрации неравновесных носителей заряда $p(x)$ будет иметь точку минимума с координатой $x_{1}$. В этой точке концентрация неравновесных носителей заряда равна $p_{1}$.

Рассмотрим условие, которое определяет характер переноса носителей зарядов вблизи перехода $\mathrm{Me}-\Pi / \Pi$, левее точки с координатой $x_{1}$. Очевидно, что, если выполняется неравенство

$$
-\left.\frac{j}{j_{d}}\left(1-\frac{j}{j_{c r}}\right) \frac{N_{d}^{2}}{p(0)^{2} L} \frac{d p}{d x}\right|_{0+}<\frac{p(0)}{L^{2}},
$$

где

$$
\left.\frac{d p}{d x}\right|_{0+} \approx-\frac{p(0)}{L^{2}}
$$

то вблизи перехода Ме-п/п будет преобладать диффузионный характер переноса носителей зарядов. Это условие эквивалентно неравенству

$$
p(0)>N_{d} \sqrt{\frac{j}{j_{d}}\left(1-\frac{j}{j_{c r}}\right)} .
$$

Если выполняется противоположное условие

$$
p(0)<N_{d} \sqrt{\frac{j}{j_{d}}\left(1-\frac{j}{j_{c r}}\right)}
$$

то уже вблизи перехода Ме-п/п будет преобладать дрейфовый характер переноса, а область с диффузионным переносом носителей зарядов будет отсутствовать. Если воспользоваться граничным условием

$$
p(0)=n_{i} \sqrt{\frac{b}{b+1} \frac{j}{j_{s 0}}}
$$

(обоснование этого граничного условия будет приведено далее в разд. 3), легко получить оценку для верхней границы плотности тока, ниже которой область с диффузионным характером переноса носителей зарядов вблизи перехода Ме-п/п будет отсутствовать:

$$
j<j_{\text {drift }}, \quad \text { где } \quad j_{\text {drift }}=j_{c r}\left[1-\frac{b}{b+1} \frac{j_{d}}{j_{s 0}}\left(\frac{n_{i}}{N_{d}}\right)^{2}\right] .
$$

Как показали дальнейшие расчеты, величина $j_{\text {drift }}$ обычно превосходит значение плотности тока $j_{s t}$. Тогда, в соответствии с результатами работ $[12,13,16]$, левее точки $x_{1}$ будет располагаться область квазинейтрального дрейфового переноса (область QND) - область 1 на на рис. 1. Правее точки $x_{1}$ будет, как в работе [12], располагаться область DSQD, обозначенная как область 2, и далее - примыкающая к $n-n^{+}$-переходу область 3, в которой реализуется диффузионный перенос неравновесных носителей заряда.

В данной работе будет рассмотрен наиболее интересный диапазон плотности тока от величины $j_{h}$, когда во всей базовой области реализуется режим ВУИ, до величины $j_{s t}$, когда область, в которой реализуется дрейфовый перенос, исчезает. Случай, когда плотность тока превышает величину $j_{s t}$, требует отдельного рассмотрения и будет представлен в дальнейших работах. 


\section{3. Вольт-амперная характеристика диода Шоттки}

Воспользуемся, как и в работах $[14,15]$, методом региональных приближений [16]. Этот метод облегчает нахождение приближенного решения нелинейного уравнения (1). В соответствии с результатами работ $[13,17]$ уравнение непрерывности в области 1 - области QND переноса - приобретает следующий вид:

$$
-\frac{j}{j_{d}}\left(1-\frac{j}{j_{c r}}\right) \frac{N_{d}^{2}}{p^{2} L} \frac{d p}{d x}=\frac{p}{L^{2}} .
$$

Эта область, как показано на рис. 1, находится между переходом Ме-п/п и точкой $x_{1}$. Отметим, что в этой области производная $d p / d x$ отрицательна.

Для нахождения решения уравнения (4) необходимо найти граничную концентрацию неравновесных носителей заряда $p(0)$. Это можно сделать, если воспользоваться граничным условием (2). После несложных преобразований, пренебрегая малой величиной $\sim\left[(b+1)^{-1}\left(N_{d} / p(0)\right)\right]$, получим следующее выражение:

$$
p(0)=p_{0}=n_{i} \sqrt{\frac{b}{b+1} \frac{j}{j_{s 0}}} .
$$

Решение дрейфового уравнения непрерывности (4) хорошо известно и приведено в классической книге [17]. В нашем случае оно имеет вид

$$
p(x)=\frac{p_{0}}{\sqrt{1+2\left(p_{0} / p^{*}\right)^{2}(x / L)}},
$$

где введено обозначение

$$
p^{*}=N_{d} \sqrt{\frac{j}{j_{d}}\left(1-\frac{j}{j_{c r}}\right)} .
$$

Из решения уравнения непрерывности (6) в области 1 легко получить протяженность этой области. Действительно, если известно, что

$$
p_{1}=p\left(x_{1}\right)
$$

где $p_{1}-$ концентрация носителей заряда на правой границе области 1 (рис. 1), тогда

$$
\frac{x_{1}}{L}=\frac{\left(p_{0} / p_{1}\right)^{2}-1}{2\left(p_{0} / p^{*}\right)^{2}}
$$

Справа от точки $x_{1}$ лежит область $x_{1}<x<x_{2}$, обозначенная на рис. 1 как область 2 . Концентрация носителей заряда справа от точки $x_{1}$ возрастает, а производная $d p / d x$ положительна. В области 2 реализуется режим DSQD, а уравнение непрерывности (1) приобретает вид

$$
\frac{d^{2} p}{d x^{2}}-\frac{j}{j_{d}}\left(1-\frac{j}{j_{c r}}\right) \frac{N_{d}^{2}}{p^{2} L} \frac{d p}{d x}=0 .
$$

В граничных точках области $2 x_{1}$ и $x_{2}$ выполняются очевидные условия

$$
\begin{aligned}
& \left.\frac{j}{j_{d}}\left(1-\frac{j}{j_{c r}}\right) \frac{N_{d}^{2}}{p_{1}^{2} L} \frac{d p}{d x}\right|_{x_{1}}=\frac{p_{1}}{L^{2}}, \\
& \left.\frac{j}{j_{d}}\left(1-\frac{j}{j_{c r}}\right) \frac{N_{d}^{2}}{p_{2}^{2} L} \frac{d p}{d x}\right|_{x_{2}}=\frac{p_{2}}{L^{2}},
\end{aligned}
$$

где $p_{2}=p\left(x_{2}\right)$.

Решение уравнения непрерывности (10) с граничными условиями (11) и (12) имеет вид

$$
\frac{p^{*}}{c L}\left(\frac{p-p_{1}}{p^{*}}+\frac{p^{*}}{c L} \ln \left|\frac{p / p^{*}-p^{*} / c L}{p_{1} / p^{*}-p^{*} / c L}\right|\right)=\frac{x-x_{1}}{L}
$$

где

$$
c=\frac{p^{*}}{L}\left[\frac{p^{*}}{p_{1}}+\left(\frac{p_{1}}{p^{*}}\right)^{3}\right] .
$$

Условие (12) совместно с (14) позволяет найти связь между концентрациями неравновесных носителей заряда в граничных точках области 2 ( $p_{1}$ и $\left.p_{2}\right)$ :

$$
\left(\frac{p_{2}}{p^{*}}\right)^{3}\left[1+\left(\frac{p^{*}}{p_{2}}\right)^{4}\right]=\frac{p^{*}}{p_{1}}\left[1+\left(\frac{p_{1}}{p^{*}}\right)^{4}\right] \approx \frac{p^{*}}{p_{1}},
$$

поскольку $\left(p_{1} / p^{*}\right)^{4} \ll 1$.

Решение уравнения непрерывности (13) и соотношение (15) позволяют выразить протяженность области 2 $\left(x_{2}-x_{1}\right)$ через величину концентрации $p_{2}$ и представить ее в виде

$$
\begin{aligned}
\frac{x_{2}-x_{1}}{L}= & {\left[\frac{p^{*} / p_{2}}{1+\left(p^{*} / p_{2}\right)^{4}}\right]^{2} } \\
& \times\left[1+4\left(p^{*} / p_{2}\right)^{4} \ln \frac{1+\left(p^{*} / p_{2}\right)^{4}}{\left(p^{*} / p_{2}\right)^{4}}\right] .
\end{aligned}
$$

Соотношение (15) позволяет представить полученную ранее протяженность области 1, описываемую выражением (9), как функцию $p_{2}$ :

$$
\frac{x_{1}}{L}=\frac{1}{2}\left\{\left(\frac{p_{2}}{p^{*}}\right)^{6}\left[1+\left(\frac{p^{*}}{p_{2}}\right)^{4}\right]^{2}-\left(\frac{p^{*}}{p_{0}}\right)^{2}\right\} .
$$

Рассмотрим область, обозначенную на рис. 1 как область 3 , ограниченную точками $x_{2}$ и $W_{n}\left(x_{2}<x<W_{n}\right)$. Высокая инжектирующая способность $n^{+}-n$-перехода обеспечивает большую концентрацию носителей заряда в этой области. Это позволяет пренебречь в уравнении непрерывности (1) дрейфовым членом по сравнению с диффузионным и рекомбинационным членами [16]. В результате в области 3 уравнение (1) приобретает диффузионный вид:

$$
\frac{d^{2} p}{d x^{2}}=\frac{p}{L^{2}}
$$


Хорошо известное общее решение уравнения (18), представляющее собой сумму нарастающей и спадающей экспонент, неудобно для дальнейшего анализа. Умножив обе части уравнения (18) на $2 d p / d x$ и понизив порядок уравнения, приведем его к виду

$$
\frac{d p}{d x}=\sqrt{c_{1}+\left(\frac{p}{L}\right)^{2}} .
$$

Неизвестную константу $c_{1}$ определим из условия (12), где производная $\left.(d p / d x)\right|_{x_{2}}$ определяется уравнением (19). В результате получим

$$
c_{1}=\left[\left(\frac{p_{2}}{p^{*}}\right)^{4}-1\right]\left(\frac{p_{2}}{L}\right)^{2} .
$$

Решение уравнения (19) с граничным условием

$$
p\left(W_{n}\right)=p_{w}
$$

имеет вид

$$
\ln \frac{p_{w}+\sqrt{c_{1} L^{2}+p_{w}^{2}}}{p+\sqrt{c_{1} L^{2}+p^{2}}}=\frac{W_{n}-x}{L} .
$$

Разрешив выражение (22) относительно $p$, легко показать, что оно приводится к стандартному диффузионному виду $p(x)=a e^{x / L}+b e^{-x / L}$.

Выражение (22) позволяет определить протяженность области 3. Действительно, при $x=x_{2}$ и $p_{2}=p\left(x_{2}\right)$ получим

$$
\begin{aligned}
& \frac{W_{n}-x_{2}}{L} \\
& =\ln \left\{\frac{p_{w}}{p_{2}} \frac{\left(p^{*} / p_{2}\right)^{2}+\sqrt{\left[1-\left(p^{*} / p_{2}\right)^{4}\right]\left(p_{2} / p_{w}\right)^{2}+\left(p^{*} / p_{2}\right)^{4}}}{1+\left(p^{*} / p_{2}\right)^{2}}\right\} .
\end{aligned}
$$

Концентрация неравновесных носителей заряда вблизи $n^{+}-n$-перехода $\left(p_{w}\right)$ определяется с помощью граничного условия (3) и имеет стандартный вид [14,15]. При больших плотностях тока, когда во всей базовой области ДШ реализуется режим ВУИ, величина $p_{w}$ хорошо аппроксимируется выражением

$$
p_{w}=n_{i} \sqrt{\frac{1}{b+1} \frac{j}{j_{s p}}},
$$

где $j_{s p}-$ ток насыщения $n^{+}-n$-перехода.

Величина $p_{2}$ - концентрация неравновесных носителей заряда на границе между областью 2 и 3 - может быть найдена численно как корень уравнения

$$
\frac{x_{1}}{L}+\frac{x_{2}-x_{1}}{L}+\frac{W_{n}-x_{2}}{L}=\frac{W_{n}}{L},
$$

где протяженности областей 1, 2 и 3 определяются соотношениями (17), (16) и (23) соответственно. Следует отметить, что определенная таким образом величина $p_{2}$ является функцией плотности тока $j$ :

$$
p_{2}=p_{2}(j)
$$

Ранее отмечалось, что с ростом плотности тока $j$ точка $x_{1}$ начинает смещаться влево, а область 1 , в которой реализуется режим кваинейтрального дрейфового переноса, уменьшается. При некоторой плотности тока $j_{s t}$ протяженность области 1 становится равной нулю и эта область исчезает. Плотность тока $j_{s t}$, при которой происходит исчезновение области 1 , может быть определена с помощью соотношения (17) из уравнения

$$
\begin{aligned}
\frac{x_{1}\left(p_{2}(j), j\right)}{L} \equiv & \frac{1}{2}\left\{\left(\frac{p_{2}(j)}{p^{*}(j)}\right)^{6}\left[1+\left(\frac{p^{*}(j)}{p_{2}(j)}\right)^{4}\right]^{2}\right. \\
& \left.-\left(\frac{p^{*}(j)}{p_{0}(j)}\right)^{2}\right\}=0 .
\end{aligned}
$$

Как было отмечено ранее, минимальная концентрация неравновесных носителей заряда $p_{1}$ достигается в точке $x_{1}$ (рис. 1). Концентрация $p_{1}$ связана с величиной $p_{2}$ соотношением (15). Необходимо помнить, что режим ВУИ реализуется во всей базовой области, если $p_{1}>N_{d}$. Поэтому, если при плотности тока, равной $j_{s t}$, величина $p_{1}$ не превосходит $N_{d}$, то область 1 исчезает до установления режима ВУИ во всей базовой области.

Найденные решения позволяют рассчитать падения напряжения на всех рассмотренных выше областях при плотностях тока, не превышающих величину $j_{s t}$. Для областей 1, 2 и 3 были получены следующие выражения:

$$
\begin{aligned}
& U_{1}=\frac{2 b}{3(b+1)^{2}} \frac{k T}{q} \frac{j L}{q D p^{*}}\left\{\left[\frac{1+\left(p^{*} / p_{2}\right)^{4}}{\left(p^{*} / p_{2}\right)^{3}}\right]^{3}-\left(p^{*} / p_{0}\right)^{3}\right\} \text {, } \\
& U_{2}=\frac{8 b}{(b+1)^{2}} \frac{k T}{q} \frac{j L}{q D p^{*}} \frac{\left(p^{*} / p_{2}\right)^{3}}{1+\left(p^{*} / p_{2}\right)^{4}} \ln \frac{1+\left(p^{*} / p_{2}\right)^{4}}{\left(p^{*} / p_{2}\right)^{4}} \text {, } \\
& U_{3}=\frac{2 b}{(b+1)^{2}} \frac{k T}{q} \frac{j L}{q D p^{*}} \frac{\left(p^{*} / p_{2}\right)^{3}}{\sqrt{1-\left(p^{*} / p_{2}\right)^{4}}} \\
& \times \ln \frac{\sqrt{1-\left(p^{*} / p_{2}\right)^{4}}+1}{\sqrt{1-\left(p^{*} / p_{2}\right)^{4}}\left(p_{2} / p_{w}\right)+}+.
\end{aligned}
$$

Здесь $p^{*}$ определяется выражением (7), а концентрация неравновесных носителей заряда в граничных точках базы ДШ, $p_{0}$ и $p_{w}$ соответственно, выражениями (5) и (24). Падение напряжения на всем базовом слое определяется суммой

$$
U_{W}=U_{1}+U_{2}+U_{3} .
$$


Полное падение напряжения на ДШ имеет стандартный вид

$$
\begin{gathered}
U_{\mathrm{Sh}}=U_{\mathrm{Me} n n^{+}}+U_{W}, \\
U_{\mathrm{Me} n n^{+}}=\frac{k T}{q} \ln \frac{p_{0} p_{w}}{n_{i}^{2}},
\end{gathered}
$$

где $U_{\mathrm{Menn^{+ }}}$ — падение напряжения на переходах $\mathrm{Me}-$ п/п и $n^{+}-n$.

На рис. 2 представлена зависимость протяженностей областей 1,2 и 3 от плотности прямого тока $j$. Расчет проводился для кремниевого ДШ со следующим набором параметров: концентрация легирующей примеси в базовой области $n$-типа проводимости $N_{d}=5 \cdot 10^{14} \mathrm{~cm}^{-3}$, толщина базового слоя $W_{n}=100$ мкм. Величина отношения $W_{n} / L$ была выбрана равной 1.5 ; плотность тока насыщения $\mathrm{Me}-n$-перехода $j_{s 0}=1 \cdot 10^{-7} \mathrm{~A} / \mathrm{cm}^{2}$, а $n^{+}-n$-перехода $j_{s p}=1 \cdot 10^{-12} \mathrm{~A} / \mathrm{cm}^{2}$.

Как видно на рис. 2, с ростом плотности прямого тока $j$ протяженность области $1\left(x_{1}-0\right)$ уменьшается и при достижении величины $j_{s t}$ область 1 исчезает. Протяженность области $2\left(x_{2}-x_{1}\right)$, как и в работах $[12,13]$, слабо зависит от плотности тока $j$. Протяженность области $3\left(W_{n}-x_{2}\right)$ с ростом плотности тока $j$ возрастает.

На рис. 3 представлена зависимость падения напряжения на областях $1,2,3$, на переходах $\mathrm{Me}-$ п/п и $n^{+}-n$ и полного падения напряжения на ДШ от плотности прямого тока $j$. Расчет проводился для ДШ с тем же набором параметров. С ростом плотности

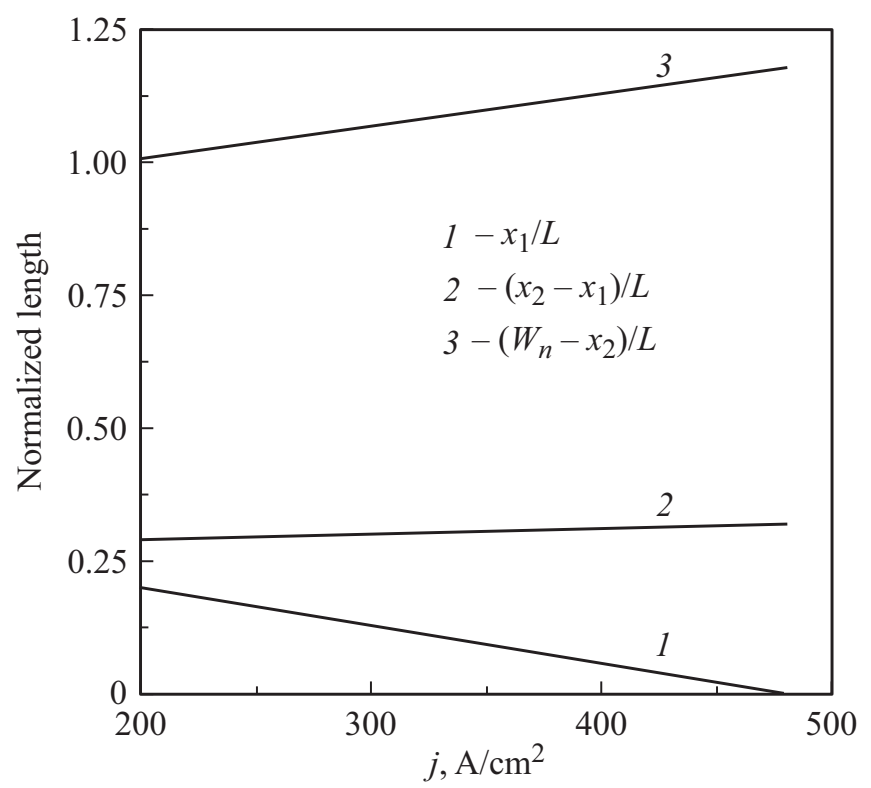

Рис. 2. Зависимости протяженностей областей 1, 2, 3 (см. рис. 1) от плотности прямого тока - расчет для кремниевого диода Шоттки при следующем наборе параметров: концентрация легирующей примеси в базовой $n$-области $N_{d}=5 \cdot 10^{14} \mathrm{~cm}^{-3}$, толщина базового слоя $W_{n}=100$ мкм, $W_{n} / L=1.5$, плотность тока насыщения контакта Ме-п/п $j_{s 0}=10^{-7} \mathrm{~A} / \mathrm{cm}^{2}$, плотность тока насыщения $n-n^{+}$-перехода $j_{s n}=10^{-12} \mathrm{~A} / \mathrm{cm}^{2}$.

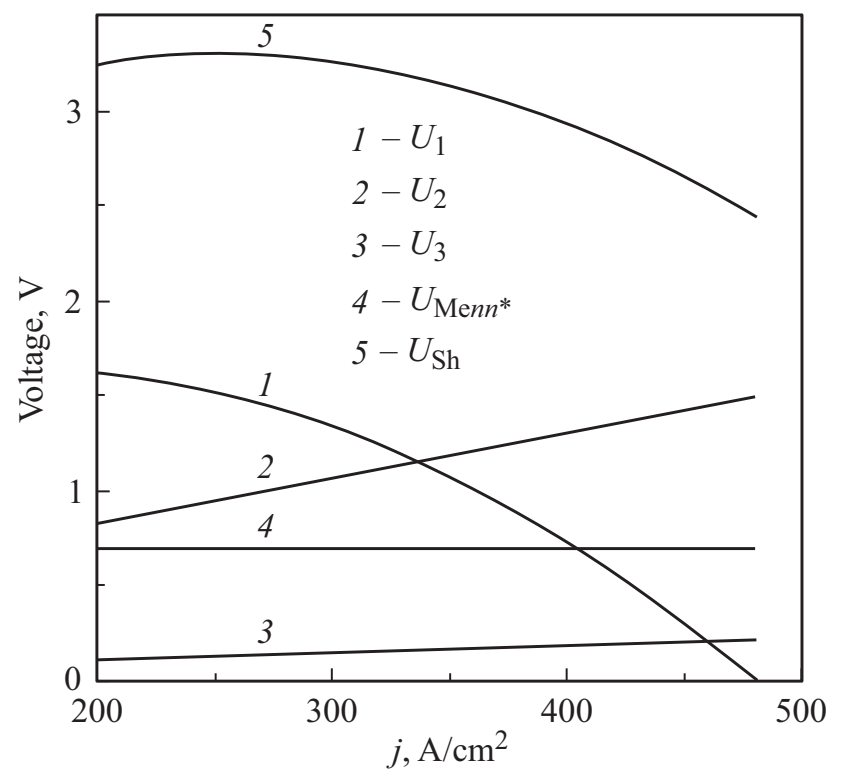

Рис. 3. Зависимости от плотности тока $j$ величин падения напряжения на области 1 (1), на области 2 (2), на области 3 (3), на контакте Ме-п/п и $n-n^{+}$-переходе (4), на всем ДШ (5).

тока $j$ падение напряжения на области 1 уменьшается и с приближением к величине $j_{s t}$ становится равным нулю. Падения напряжения на области 2 и на области 3 увеличиваются. Падение напряжения на переходах $\mathrm{Me}-n$ и $n^{+}-n$ с ростом плотности тока изменяется логарифмически слабо. Полное падение напряжения на ДШ с ростом плотности тока $j$ изменяется немонотонно и при некоторой плотности тока имеет точку максимума. Немонотонный характер зависимости полного падения напряжения на ДШ $U_{\mathrm{Sh}}$ от плотности тока $j$ приводил к появлению на ВАХ ДШ участка с отрицательным дифференциальным сопротивлением $R_{d}$.

\section{4. Сравнение с численным экспериментом}

Для проверки результатов, полученных с помощью рассмотренной выше модели ВАХ ДШ, был проведен численный эксперимент с помощью программы INVESTIGATION. Подробное описание этой программы приведено в работах $[18,19]$. Работа этой программы основана на численном решении уравнений непрерывности для электронов и дырок и уравнения Пуассона. При решении учитывались нелинейные эффекты, обусловленные ВУИ в базовом слое и сильным легированием эмиттерной области. Расчет проводился для кремниевого ДШ со следующим набором параметров: толщина базового слоя $n$-типа проводимости $W_{n}=100$ мкм, толщина эмиттерного $n^{+}$-слоя 20 мкм, концентрация донорной примеси в базовом слое $N_{d}=5 \cdot 10^{14} \mathrm{~cm}^{-3}$, концентрация донорной примеси в эмиттере $N_{d}^{+}=1 \cdot 10^{19} \mathrm{~cm}^{-3}$. Высота барьера Шоттки для электронов равнялась 
$\varphi_{B n}=0.85$ эВ. Были выбраны следующие значения времен жизни неравновесных носителей заряда при ВУИ: $\tau=2.1$ (1), 2.8 (2), 3.8 (3), 5.4 (4), 8.5 (5), 15.1 мкс (6). Этим значениям соответствовали следующие величины отношения $W_{n} / L: W_{n} / L=1.6(1), 1.4(2), 1.2(3), 1.0$ (4), $0.8(5), 0.6(6)$.

Результаты расчета ВАX ДШ представлены на рис. 4. Расчет проводился в широком диапазоне плотностей тока, так как использовавшаяся программа INVESTIGATION не накладывает ограничений на величину уровня инжекции в базовой области. Как видно из рис. 4, при увеличении времени жизни неравновесных носителей заряда $\tau$ и соответствуюшем уменьшении величины отношения $W_{n} / L S$-образность ВАХ сначала уменьшается, а затем пропадает совсем.

На рис. 5 приведены ВАХ ДШ, рассчитанные в соответствии с представленной выше моделью. Расчет проводился для структур с тем же набором параметров. Из рис. 5 видно, что характер зависимости ВАХ от величины отношения $W_{n} / L$ качественно такой же, как на рис. 4. При уменышении величины отношения $W_{n} / L$ также наблюдалось уменьшение $S$-образности ВАХ. Как видно из рис. 5 , уменьшение величины отношения $W_{n} / L$ приводит к уменьшению величины плотности тока $j_{s t}$. Физически это объясняется уменьшением рекомбинационных потерь неравновесных носителей заряда при уменьшении отношения $W_{n} / L$ и увеличением концентрации неравновесных носителей заряда вблизи перехода $\mathrm{Me}-$ п/п. Это приводит к уменьшению электрического поля, необходимого для поддержания в области, прилегающей к переходу $\mathrm{Me}-\Pi / п$, тока с требуемой плот-

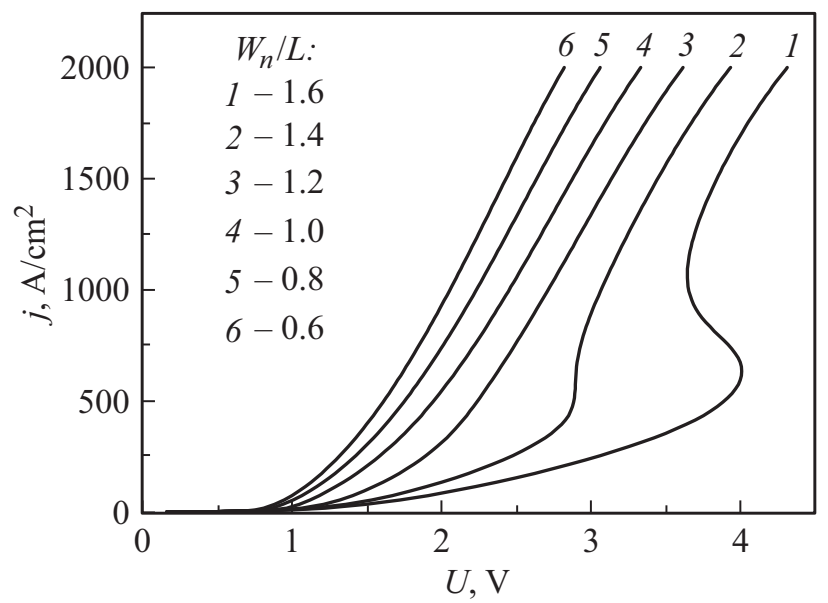

Рис. 4. Результаты численного расчета ВАХ ДШ с помощью программы „INVESTIGATION“ при следующих значениях параметров структуры: толщина базового $n$-слоя $W_{n}=100$ мкм, толщина эмиттерного $n^{+}$-слоя $W_{n}=20$ мкм, концентрация доноров в $n$-базе $N_{d}=5 \cdot 10^{14} \mathrm{~cm}^{-3}$, концентрация доноров в $n^{+}$-эмиттере $N_{d}^{+}=1 \cdot 10^{19} \mathrm{~cm}^{-3}$, высота барьера Шоттки для электронов $\varphi_{B n}=0.85$ эВ. Выбраны следующие значения времени жизни неравновесных носителей заряда при высоком уровне инжекции: $\tau=2.1(1), 2.8(2), 3.8$ (3), $5.4(4), 8.5$ (5), 15.1 мкс (6).

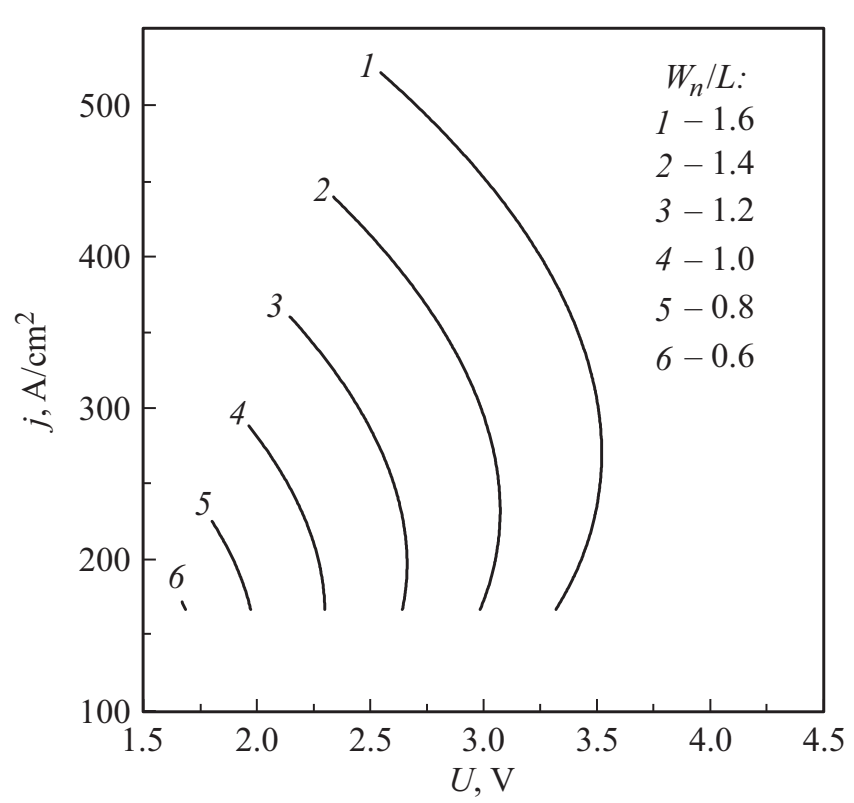

Рис. 5. ВАХ ДШ, рассчитанные с помощью описанной аналитической модели. Расчет проводился при тех же значениях электрофизических параметров, что на рис. 4. Характер изменения ВАХ от величины отношения $W_{n} / L$ качественно такой же, как на рис. 4.

ностью $j$ и соответствующего уменьшения областей, в которых реализуются режимы QND и DSQD. При этом точка минимума на распределении концентрации неравновесных носителей заряда сдвигается в сторону перехода $\mathrm{Me}-$ п/п. По этой же причине уменьшается плотность тока $j_{s t}$, при которой происходит исчезновение области 1.

Сравнение данных, представленных на рис. 4 и 5, показывает заметные количественные различия, полученные при численных и аналитических расчетах. Эти различия связаны с погрешностью метода региональных приближений [16]. Однако с точки зрения качественного поведения BAX ДШ они подтверждают правильность основного вывода аналитической модели. Уменьшение величины отношения $W_{n} / L$ приводит к уменьшению и исчезновению области, в которой реализуется квазинейтральный дрейфовый режим переноса неравновесных носителей заряда. В результате наблюдается уменьшение и исчезновение $S$-образности ВАХ ДШ при ВУИ в базовой области.

\section{5. Заключение}

В работе была предложена модель для расчета ВАХ ДШ при ВУИ в базовой области для произвольной величины отношения $W_{n} / L$. При разработке модели было использовано граничное условие для перехода Ме-п/п, которое ранее успешно применялось в работах $[14,15]$. Это граничное условие учитывает слабую инжектирующую способность контакта Шоттки. 
В работе показано, что, если $W_{n} / L>1$, наблюдается $S$-образная ВАХ ДШ при ВУИ в базовой области. Это объясняется появлением вблизи перхода Ме-п/п области, в которой реализуется квазинейтральный дрейфовый перенос носителей заряда. В этой же области электрическое поле достигает наибольшего значения. С ростом плотности тока $j$ эта область уменьшается и при достижении величины $j_{s t}$ исчезает. Это обусловливает появление на ВАХ ДШ участка с отрицательным дифференциальным сопротивлением. При уменьшении величины отношения $W_{n} / L$ протяженность области, в которой реализуется квазинейтральный дрейфовый перенос носителей заряда, уменьшается, и при некоторой величине отношения $W_{n} / L$ эта область не возникает.

Результаты, полученные с помощью аналитической модели, были подтверждены численным экспериментом, выполненным с помощью программы INVESTIGATION.

\section{Конфликт интересов}

Авторы заявляют, что у них нет конфликта интересов.

\section{Список литературы}

[1] L.M. Hillkirk. Solid State Electron., 48, 2181 (2004).

[2] M.E. Levinshtein, T.T. Mnatsakanov, P.A. Ivanov, J.W. Palmour, M.K. Das, B.A. Hull. Semicond. Sci. Technol., 23, 085011 (2008).

[3] J.W. Palmour, M.E. Levinshtein, P.A. Ivanov, Q.J. Zhang. J. Phys. D: Appl. Phys., 48, 235103 (2015).

[4] D.L. Scharfetter. Solid State Electron., 8, 299 (1965).

[5] A. Yu, E. Snow. Solid State Electron., 12, 155 (1969).

[6] B. Elfsten, P.A. Tove. Solid State Electron., 28, 721 (1985).

[7] W.T. Ng, S. Liang, C.A.T. Salama. Solid State Electron., 33, 39 (1990).

[8] K. Sarpatwari, S.E. Mohney, S. Ashok, O.O. Awadelkarim. Phys. Status Solidi A, 207, 1509 (2010).

[9] R. Singh, D.C. Capell, A.R. Hefner, J. Lai, J.W. Palmour. IEEE Trans. Electron Dev., 49, 2054 (2002).

[10] J.H. Zhao, K. Sheng, R.C. Lebron-Velilla. In: SiC Materials and Devices, v. 1, ed. by M. Shur, S. Rumyantsev, M. Levinshtein (World Scientific, Singapore-New Jersey, 2006) p. 117.

[11] C. Buttay, C. Raynaud, H. Morel, G. Civrac, M.-L. Locatelli, F. Morel. IEEE Trans. Electron Dev., 59, 761 (2012).

[12] T.T. Mnatsakanov, M.E. Levinshtein, A.G. Tandoev, S.N. Yurkov. J. Appl. Phys., 105, 044506 (2009).

[13] T.T. Mnatsakanov, A.G. Tandoev, M.E. Levinshtein, S.N. Yurkov. Semicond. Sci. Technol., 24, 075006 (2009).

[14] T.T. Mnatsakanov, M.E. Levinshtein, A.G. Tandoev, S.N. Yurkov, J.W. Palmour. Solid State Electron., 121, 41 (2016).

[15] T.T. Mnatsakanov, A.G. Tandoev, M.E. Levinshtein, S.N. Yurkov, J.W. Palmour. Semiconductors, 51, 8 (2017).

[16] M. Lampert, P. Mark. Current injection in solids (Academic Press, N.Y.-London, 1970).

[17] V.B. Shuman, T.T. Mnatsakanov, M.E. Levinshtein, A.G. Tandoev, S.N. Yurkov, J.W. Palmour. Semicond. Sci. Technol., 26, 085016 (2011).

[18] T.T. Mnatsakanov, I.L. Rostovtsev, N.I. Philatov. Solid State Electron., 30, 579 (1987).
[19] T.T. Mnatsakanov. Phys. Status Solidi B, 143, 225 (1987).

Редактор Л.В. Шаронова

\section{$S$-type current-voltage characteristics of Schottky diodes at high current densities}

\section{A.G. Tandoev, T.T. Mnatsakanov, S.N. Yurkov}

Moscow Power Engineering Institute, 111250 Moscow, Russia

\begin{abstract}
A consistent analysis of the effect of different charge carrier quasyneutral transport regimes on characteristics of semiconductor structures is presented in the paper. In addition to the commonly accepted diffusion and quasineutral drift regimes, a recently found regime of diffusion stimulated by quasineutral drift (DSQD) has been also taken into account. The charge carrier transport in Schottky diode has been examined in detail. The results of analytical study have been confirmed using numerical modeling.
\end{abstract}

